\section{(1)}

CrossMark

\title{
Analysis of loss to follow-up in 4099 multidrug-resistant pulmonary tuberculosis patients
}

\author{
Ian F. Walker (101, Oumin Shi ${ }^{2,3}$, Joseph P. Hicks ${ }^{1}$, Helen Elsey ${ }^{1}$, Xiaolin Wei (1) ${ }^{2}$, \\ Dick Menzies ${ }^{4}$, Zhiyi Lan ${ }^{4}$, Dennis Falzon ${ }^{5}$, Giovanni Battista Migliori (106, \\ Carlos Pérez-Guzmán ${ }^{7,8}$, Mario H. Vargas ${ }^{8,9}$, Lourdes García-García ${ }^{10}$, \\ José Sifuentes Osornio ${ }^{11}$, Alfredo Ponce-De-León ${ }^{12}$, Martie van der Walt ${ }^{13}$ and \\ James N. Newell ${ }^{1}$
}

Affiliations: ${ }^{1}$ Nuffield Centre for International Health and Development, University of Leeds, Leeds, UK. ${ }^{2}$ Dalla Lana School of Public Health, University of Toronto, Toronto, ON, Canada. ${ }^{3}$ Shenzhen Second People's Hospital, Shenzhen University, Shenzhen, China. "Montreal Chest Institute, McGill International TB Centre, McGill University, Montreal, QC, Canada. ${ }^{5}$ Global TB Programme, World Health Organization, Geneva, Switzerland. ${ }^{6}$ Instituti Clinici Scientifici Maugeri IRCCS, Tradate, Italy. ${ }^{7}$ Hospital General Tercer Milenio, Aguascalientes, Mexico. ${ }^{8}$ Instituto Mexicano del Seguro Social, Mexico City and Aguascalientes, Mexico. ${ }^{9}$ Instituto Nacional de Enfermedades Respiratorias, Mexico City, Mexico. ${ }^{10}$ Instituto Nacional de Salud Pública, Cuernavaca, Mexico. ${ }^{11}$ Dirección de Medicina Interna, Instituto Nacional de Ciencias Médicas y Nutrición Salvador Zubirán, Mexico City, Mexico. ${ }^{12}$ Laboratorio Nacional de Máxima Seguridad Biológica y Microbiología Clínica, Departamento de Infectología, Instituto Nacional de Ciencias Médicas y Nutrición Salvador Zubirán, Mexico City, Mexico. ${ }^{13}$ South African Medical Research Council, Tygerberg, South Africa.

Correspondence: lan F. Walker, Nuffield Centre for International Health and Development, University of Leeds, Room 1031, Worsley Building, Clarendon Way, Leeds, LS2 9NL, UK. E-mail: i.walkeraleeds.ac.uk

@ERSpublications

Globally, 75\% of loss to follow-up occurs in the first 11 months of MDR-TB treatment. Highest risk is HIV positive, age 36-50 years and standardised regimen. http://bit.ly/2GwKcS1

Cite this article as: Walker IF, Shi O, Hicks JP, et al. Analysis of loss to follow-up in 4099 multidrugresistant pulmonary tuberculosis patients. Eur Respir J 2019; 54: 1800353 [https://doi.org/10.1183/ 13993003.00353-2018].

ABSTRACT Loss to follow-up (LFU) of $\geqslant 2$ consecutive months contributes to the poor levels of treatment success in multidrug-resistant tuberculosis (MDR-TB) reported by TB programmes. We explored the timing of when LFU occurs by month of MDR-TB treatment and identified patient-level risk factors associated with LFU.

We analysed a dataset of individual MDR-TB patient data (4099 patients from 22 countries). We used Kaplan-Meier survival curves to plot time to LFU and a Cox proportional hazards model to explore the association of potential risk factors with LFU.

Around one-sixth $(\mathrm{n}=702)$ of patients were recorded as LFU. Median (interquartile range) time to LFU was 7 (3-11) months. The majority of LFU occurred in the initial phase of treatment (75\% in the first 11 months). Major risk factors associated with LFU were: age 36-50 years (HR 1.3, 95\% CI 1.0-1.6; $\mathrm{p}=0.04$ ) compared with age $0-25$ years, being HIV positive (HR 1.8, 95\% CI 1.2-2.7; $\mathrm{p}<0.01$ ) compared with HIV negative, on an individualised treatment regimen (HR 0.7, 95\% CI 0.6-1.0; $\mathrm{p}=0.03$ ) compared with a standardised regimen and a recorded serious adverse event (HR 0.5, 95\% CI 0.4-0.6; $\mathrm{p}<0.01$ ) compared with no serious adverse event.

Both patient- and regimen-related factors were associated with LFU, which may guide interventions to improve treatment adherence, particularly in the first 11 months. 


\section{Introduction}

Multidrug-resistant tuberculosis (MDR-TB) is a growing challenge to TB treatment programmes globally. Treatment completion rates remain low at 50\%. A substantial proportion of MDR-TB patients are considered "loss to follow-up" (LFU; previously termed "treatment default") [1-4], defined as those "whose treatment was interrupted for 2 consecutive months or more" [4]. Global estimates of LFU are only available for the past decade, showing only modest reductions over time. The latest estimate is $15 \%$ of those starting treatment being lost and a further $7 \%$ with no outcome information recorded, many of whom in reality are likely to be LFU [5]. Patients who do not complete pulmonary MDR-TB treatment pose a public health risk of ongoing transmission of resistant, infectious disease and have a high likelihood of death.

Current evidence from recent MDR-TB treatment cohorts identifies that LFU is associated with being male [6], substance misuse [6-11], resistance to a high number of anti-TB drugs [6,12, 13], absence of early culture conversion $[6,11,13,14]$, poor patient-provider relationships $[8,10]$, greater disease severity [7], HIV co-infection [15] and occurrence of drug side-effects [10, 13]. However, there is a lack of conclusive evidence globally on the timing of when LFU occurs during treatment.

Several recent studies suggest that the majority of LFU may take place in the early stages of MDR-TB treatment. The percentage of total LFU that occurred in the intensive phase (first 6-8 months) of MDR-TB treatment was $78 \%$ in the Philippines (71 out of 91 LFU patients) [10], 71\% in Armenia (69 out of 97 LFU patients) [13], 73\% in Pakistan (24 out of 33 LFU patients) [16] and 41\% in Georgia (187 out of 458 LFU patients) [11]. The total LFU occurring by 6 months was $87 \%$ in India (133 out of 153 LFU patients) [14]. In Uzbekistan, median time to LFU was 6 months ( $\mathrm{n}=710, \mathrm{LFU}=142)$ [12].

To optimise the management of MDR-TB patients and limit the numbers who become LFU, national TB treatment programmes would benefit from knowing programmatic and individual characteristics associated with LFU and when it is likely to occur. Interventions targeted at individuals with these risk factors at these time-points could reduce rates of LFU and ultimately assist in controlling the epidemic. Analysis to specify the time of LFU more accurately would optimise the timing of such interventions. Given the dearth of knowledge on this topic, here we are able to use the largest ever multicountry, individual MDR-TB dataset to identify the timing of LFU in the treatment of pulmonary MDR-TB, and to identify programmatic and patient-level risk factors associated with LFU [1-3].

\section{Methods}

The dataset of the Collaborative Group for Meta-Analysis of Individual Patient Data in MDR-TB was used for this analysis [1]. This dataset includes individual-level treatment data from 9562 pulmonary MDR-TB patients from TB clinics or programmes from 23 countries, reported in 32 previously published studies. It was originally developed to ascertain optimum treatment regimens by using patient-level data. An aggregated dataset for meta-analysis was created from data using variables related to this purpose from study authors of these published cohort studies. Although the data are now $>10$ years old, they retain their relevance today as unacceptably high rates of LFU persist. Although some aspects of MDR-TB treatment have developed over the past 10 years, there are enough fundamental similarities that enable this large dataset to provide valuable findings from the recent past to inform current treatment provision.

Patients had to have received at least 1 month of treatment in order to be included in the original individual patient data dataset. The patient characteristics and definitions of the variables within the dataset have been described elsewhere [1]. Cohorts in the original study were not included if the lead investigator did not give permission to include their data or if they only reported planned duration rather than actual treatment duration at the individual level.

For our analysis, patients were included if they were alive at the point of LFU. Patients were defined as LFU if their outcome was recorded as "defaulted", "transferred out (with unknown outcome)" or "unknown" in the dataset, based on the outcome definitions available at the time data were collected [17]. Time of LFU for each patient was identified by their recorded duration of treatment (in months). The treatment cohorts included in this analysis used a variety of drug regimens and treatment lengths; most were 20-24 months. Those with a duration of treatment $>24$ months were truncated at 24 months. Records were excluded if there were no data on duration of treatment (missing data). We also excluded patient records whose outcome was death. It is possible that a good number of these patients may have chosen to stop MDR-TB treatment before death. We were concerned that retaining patients who died would introduce more bias than if we removed them and so we decided to exclude all patients who died.

We identified the independent variables that were available from the dataset to include in the analysis, informed from previous studies where significant associations with LFU had been identified (see Introduction) [6-13]. Variables included in the analysis were: age, sex, HIV co-infection status, extensive 
TB disease (defined as acid-fast bacilli (AFB) smear positive or cavities on chest radiography if no information about AFB smear was available), type of regimen (standardised versus individualised), previous TB therapy (defined as previous treatment with first-line drugs or with second-line TB drugs for $\geqslant 1$ month), recorded drug resistance to pyrazinamide, ethambutol and streptomycin (in addition to isoniazid and rifampicin), recorded serious adverse events (that required a change in therapy) and AFB smear status. We were also interested in variations by national income, and so we distinguished study cohorts from high-income and middle-income countries within the analysis (according to World Bank classifications) [18]. There were no cohorts from low-income countries.

The proportions of all LFU patients are reported. As the data were negatively skewed we used quantile regression analysis to compare median time to LFU for subgroups (per independent variable). We then employed Kaplan-Meier methods to plot survival curves and estimate the unadjusted time to LFU across the treatment period. To explore this distribution further, we undertook sensitivity analysis by plotting timing of LFU by middle-income and high-income countries. Lastly, a Cox proportional hazards model was created to assess the effects of potential risk factors on LFU. First, unadjusted and then adjusted hazard ratios were calculated (including all variables within the Cox model) with $95 \%$ confidence intervals and the associated (two-sided) p-values. We checked the assumptions of the proportional hazards model using the empirical score process that compares observed with simulated processes to detect violations. Patient-level data were considered to be clustered within study cohorts and so the model used a random effects, multilevel analysis to account for this. SAS version 9.4 (SAS Institute, Cary, NC, USA) was used to undertake the statistical analysis.

The original ethics approval for the analysis of anonymised data for the Collaborative Group for Meta-Analysis of Individual Patient Data also covered this secondary analysis and therefore no separate ethics review was needed [1].

\section{Results}

Data originated from 29 studies in 22 countries: nine studies were from Asia (four of which were from South Korea), eight from North America, four from Europe, two from South America, four from former Soviet states, one from South Africa, and one from Iran (see supplementary material). The exclusions from the full dataset $(n=9562)$ were one treatment cohort from Bangladesh without permission to include $(\mathrm{n}=607)$, two treatment cohorts from South Africa and Italy that reported planned rather than actual duration of treatment $(n=2204$ and $n=95$, respectively), and records with no follow-up time recorded $(n=1742)$. Of the included cohorts, patients with an outcome of death $(n=815)$ were then excluded. Our dataset therefore included 4099 patients, of which 702 (17.1\%) were recorded as LFU. The characteristics of the patients in our dataset are described in table 1.

\section{Timing of LFU}

After 6 months of treatment, $48 \%(n=336)$ of those who were recorded as LFU had been lost (figure 1). At the end of the intensive stage of treatment at 8 months, $64 \%(n=448)$ had been lost. By the 12 -month stage, $81 \%(n=567)$ had been lost and by 18 months it was $95 \%(n=669)$.

For all patients recorded as LFU, the median (interquartile range (IQR)) time to LFU was 7 (3-11) months. The median time to LFU was broadly similar when analysed by subgroups (table 1).

The rate of LFU occurrence was higher in the initial stage of treatment (first 6-8 months) than the continuation stage and flattened off considerably after 18 months of treatment (figures 1 and 2). Our analysis of LFU by country national income indicated very similar probability of LFU throughout treatment (figure 3).

\section{Risk factors associated with LFU}

After graphical and statistical checks, we were satisfied the assumptions of our Cox proportional hazards model held. After adjusting for all other variables in this model, several risk factors were significantly associated with LFU (table 1 and figure 4). Those aged 36-50 years had 27\% higher incidence of LFU (HR 1.3, 95\% CI 1.0-1.6; $\mathrm{p}=0.04$ ) compared with those aged $0-25$ years. Those with HIV had $82 \%$ higher incidence of LFU (HR 1.8, 95\% CI 1.2-2.7; p<0.01) compared with HIV-negative patients. Those receiving an individualised treatment regimen had $26 \%$ lower incidence of LFU (HR 0.7, 95\% CI 0.6-1.0; p=0.03) compared with a standardised regimen. Those who had a recorded serious adverse event during treatment had a $51 \%$ lower incidence of LFU (HR 0.5, 95\% CI 0.4-0.6; $\mathrm{p}<0.01)$ compared with no serious adverse event. 
TABLE 1 Characteristics of multidrug-resistant tuberculosis (TB) patients and risk factors associated with time to loss to follow-up (LFU)

\begin{tabular}{|c|c|c|c|c|c|c|c|c|c|}
\hline \multirow[t]{2}{*}{ Variable } & \multicolumn{4}{|c|}{ Patient characteristics } & \multirow{2}{*}{$\begin{array}{l}\text { Quantile regression } \\
\text { analysis: median (IQR) } \\
\text { time to LFU months }\end{array}$} & \multicolumn{4}{|c|}{ Cox proportional hazards analysis } \\
\hline & Category & $\begin{array}{l}\text { Patients in group } n \\
\text { ( } \% \text { of total patients) }\end{array}$ & $\begin{array}{l}\text { Recorded as LFU } \\
\mathrm{n}(\% \text { of total LFU })\end{array}$ & $\begin{array}{l}\text { LFU in each } \\
\text { category } \%\end{array}$ & & $\begin{array}{c}\text { Unadjusted HR } \\
(95 \% \mathrm{CI})\end{array}$ & p-value & $\begin{array}{c}\text { Adjusted HR } \\
(95 \% \mathrm{CI})\end{array}$ & p-value \\
\hline Total & All patients & 4099 & 702 & 17.1 & $7(3-11)$ & & & & \\
\hline \multirow[t]{2}{*}{ Sex } & Female & $1249(30.5)$ & $181(25.8)$ & 14.5 & $7(4-11)$ & Reference & & Reference & \\
\hline & Male & $2850(69.5)$ & $521(74.2)$ & 18.3 & $7(3-11)$ & $1.3(1.1-1.6)$ & $<0.01$ & $1.2(1.0-1.4)$ & 0.06 \\
\hline \multirow[t]{4}{*}{ Age } & $0-25$ years & 735 (17.9) & $104(14.8)$ & 14.1 & $7(4-13)$ & Reference & & Reference & \\
\hline & $26-35$ years & $1148(28.0)$ & $178(25.4)$ & 15.5 & $7(3-12)$ & $1.2(0.9-1.5)$ & 0.22 & $1.1(0.8-1.4)$ & 0.62 \\
\hline & $36-50$ years & $1377(33.6)$ & $269(38.3)$ & 19.5 & $7(4-11)$ & $1.5(1.2-1.9)$ & $<0.01$ & $1.3(1.0-1.6)$ & 0.04 \\
\hline & $51-99$ years & $839(20.5)$ & $151(21.5)$ & 18.0 & $6(3-11)$ & $1.4(1.1-1.8)$ & 0.01 & $1.3(1.0-1.6)$ & 0.08 \\
\hline \multirow[t]{2}{*}{ National income category } & High income & 2184 (53.3) & 374 (53.3) & 17.1 & $7(4-12)$ & Reference & & Reference & \\
\hline & Middle income & 1915 (46.7) & 328 (46.7) & 17.1 & $6(2-11)$ & $1.1(0.9-1.2)$ & 0.37 & $0.9(0.7-1.2)$ & 0.48 \\
\hline \multirow[t]{3}{*}{ HIV status } & Negative & 3644 (88.9) & 599 (85.3) & 16.4 & $7(4-11)$ & Reference & & Reference & \\
\hline & Positive & $111(2.7)$ & $32(4.6)$ & 28.8 & $8(4-15)$ & $1.8(1.3-2.5)$ & $<0.01$ & $1.8(1.2-2.7)$ & $<0.01$ \\
\hline & Unknown & 344 (8.4) & $71(10.1)$ & 20.6 & $7(3-11)$ & $1.3(1.0-1.6)$ & 0.05 & $1.6(1.2-2.0)$ & $<0.01$ \\
\hline \multirow[t]{3}{*}{ Extensive TB disease } & No & 1022 (24.9) & $161(22.9)$ & 15.8 & $6(3-11)$ & Reference & & Reference & \\
\hline & Yes & $3019(73.7)$ & $535(76.2)$ & 17.7 & $7(4-11)$ & $1.1(1.0-1.4)$ & 0.16 & $1.0(0.7-1.5)$ & 0.99 \\
\hline & Unknown & $58(1.4)$ & $6(0.9)$ & 10.3 & $9(1-17)$ & $0.6(0.3-1.4)$ & 0.26 & $0.9(0.4-2.1)$ & 0.74 \\
\hline \multirow[t]{2}{*}{ TB treatment regimen } & Standardised & $377(9.2)$ & $98(14.0)$ & 26.0 & $7(3-11)$ & Reference & & Reference & \\
\hline & Individualised & 3722 (90.8) & $604(86.0)$ & 16.2 & $8(6-12)$ & $0.5(0.4-0.6)$ & $<0.01$ & $0.7(0.6-1.0)$ & 0.03 \\
\hline \multirow[t]{4}{*}{ Previous TB drug treatment } & None & 965 (23.5) & $168(23.9)$ & 17.4 & $7(4-12)$ & Reference & & Reference & \\
\hline & First line & $2073(50.6)$ & $356(50.7)$ & 17.2 & $7(3-10)$ & $0.8(0.7-1.1)$ & 0.96 & $0.8(0.7-1.0)$ & 0.08 \\
\hline & Second line & $653(15.9)$ & $106(15.1)$ & 16.2 & $7(3-13)$ & $1.0(0.8-1.6)$ & 0.15 & $0.9(0.7-1.2)$ & 0.53 \\
\hline & Unknown & $378(9.2)$ & $72(10.3)$ & 19.0 & $7(4-14)$ & $1.2(0.9-1.6)$ & 0.17 & $0.8(0.6-1.2)$ & 0.50 \\
\hline \multirow[t]{3}{*}{ Resistance to pyrazinamide } & Susceptible & 1135 (27.7) & $174(24.8)$ & 15.3 & $8(5-12)$ & Reference & & Reference & \\
\hline & Resistant & $1561(38.1)$ & 235 (33.5) & 15.1 & $7(5-12)$ & $0.98(0.8-1.2)$ & 0.82 & $1.1(0.9-1.3)$ & 0.59 \\
\hline & Unknown & 1403 (34.2) & $293(41.7)$ & 20.9 & $6(2-10)$ & $1.7(1.4-2.0)$ & $<0.01$ & $2.4(1.9-3.0)$ & $<0.01$ \\
\hline \multirow[t]{3}{*}{ Resistance to ethambutol } & Susceptible & 1386 (33.8) & $258(36.8)$ & 18.6 & $6(3-10)$ & Reference & & Reference & \\
\hline & Resistant & $2241(54.7)$ & $362(51.6)$ & 16.2 & $7(4-12)$ & $0.8(0.7-1.0)$ & 0.01 & $0.9(0.8-1.1)$ & 0.51 \\
\hline & Unknown & 472 (11.5) & $82(11.7)$ & 17.4 & $8(6-15)$ & $0.8(0.6-1.1)$ & 0.11 & $1.1(0.8-1.6)$ & 0.51 \\
\hline \multirow[t]{3}{*}{ Resistance to streptomycin } & Susceptible & $967(23.6)$ & $195(27.8)$ & 20.2 & $7(4-12)$ & Reference & & Reference & \\
\hline & Resistant & $2831(69.1)$ & 481 (68.5) & 17.0 & $7(3-11)$ & $0.9(0.8-1.1)$ & 0.18 & $0.9(0.8-1.1)$ & 0.37 \\
\hline & Unknown & $301(7.3)$ & $26(3.7)$ & 8.6 & $13(6-16)$ & $0.4(0.3-0.6)$ & $<0.01$ & $0.2(0.1-0.4)$ & $<0.01$ \\
\hline \multirow[t]{3}{*}{ Serious adverse event } & No & $814(19.9)$ & $210(29.9)$ & 25.8 & $6(1-10)$ & Reference & & Reference & \\
\hline & Yes & $960(23.4)$ & $134(19.1)$ & 14.0 & $8(5-11)$ & $0.5(0.4-0.6)$ & $<0.01$ & $0.5(0.4-0.6)$ & $<0.01$ \\
\hline & Unknown & 2325 (56.7) & $358(51.0)$ & 15.4 & $7(4-12)$ & $0.5(0.4-0.6)$ & $<0.01$ & $0.5(0.4-0.7)$ & $<0.01$ \\
\hline \multirow[t]{3}{*}{ AFB smear } & Negative & $732(17.9)$ & $124(17.7)$ & 16.9 & $6(3-13)$ & Reference & & Reference & \\
\hline & Positive & 2412 (58.8) & $457(65.1)$ & 18.9 & $7(3-12)$ & $1.1(0.9-1.4)$ & 0.17 & $1.0(0.7-1.7)$ & 0.84 \\
\hline & Unknown & 955 (23.3) & $121(17.2)$ & 12.7 & $7(4-10)$ & $0.71(0.5-0.9)$ & $<0.01$ & $0.9(0.6-1.3)$ & 0.54 \\
\hline
\end{tabular}


FIGURE 1 Cumulative percentage of loss to follow-up (LFU) for all multidrug-resistant tuberculosis (MDR-TB) patients and for LFU patients only by month of treatment. Note that the two sets of data points show the timing of LFU among all MDR-TB patients $(n=4099)$ and for those that were recorded as LFU ( $n=702)$.

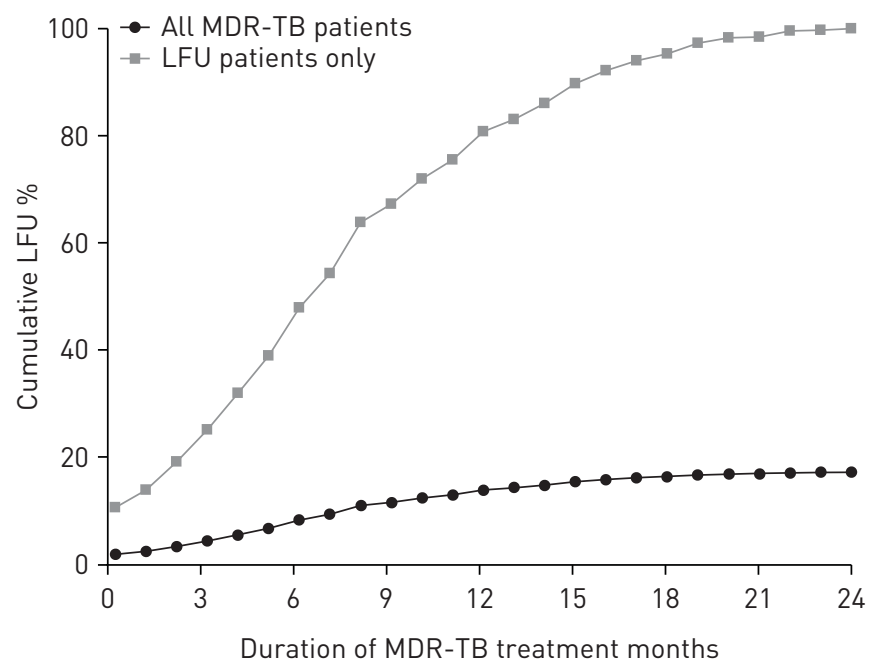

\section{Discussion}

Incomplete treatment of MDR-TB is an important driver of continued transmission and avoidable $\mathrm{TB}$ morbidity and mortality worldwide. Using the largest dataset of individual MDR-TB patients currently available, our study is the most comprehensive assessment to date of the timing and risk factors for LFU. The overall frequency of $17.1 \%$ is slightly lower than reported in the full dataset (23\%) [1] and much lower when deaths are excluded from global TB monitoring at the time $(28.6 \%)[5,19]$. This substantial difference is likely to be due to the countries included in our study. Of note, large numbers of MDR-TB patients in India, China, Kazakhstan and one cohort from South Africa are not included, and this may limit the generalisability of our findings The median (IQR) time to LFU was 7 (3-11) months. Subgroups with a higher risk of LFU were those aged 36-50 years (versus 0-25 years), those HIV positive (versus HIV negative), those receiving a standardised treatment regimen (versus an individualised regimen) and those with no reported serious adverse event (versus reported serious adverse event). The majority of LFU occurred in the initial stage of treatment, with $75 \%$ of all LFU occurring in the first 11 months of treatment.

Our results demonstrate that individualised treatment regimens may prevent patients from becoming LFU, although it should be noted that the definition of standardised regimens has changed over recent decades and so this may not be a consistent comparison across cohorts. An individualised approach is challenging to scale-up in many settings as it depends on robust drug sensitivity testing and the clinical skills to alter regimens accordingly. From a programmatic perspective, the policy decision about which model of treatment to pursue should consider LFU as a key factor. This may also have relevance for the scale-up of standardised 9-11-month regimens that the World Health Organization now promotes [20].

FIGURE 2 Time to loss to follow-up (LFU) after starting multidrugresistant tuberculosis (MDR-TB) treatment for all patients, using Kaplan-Meier analysis.

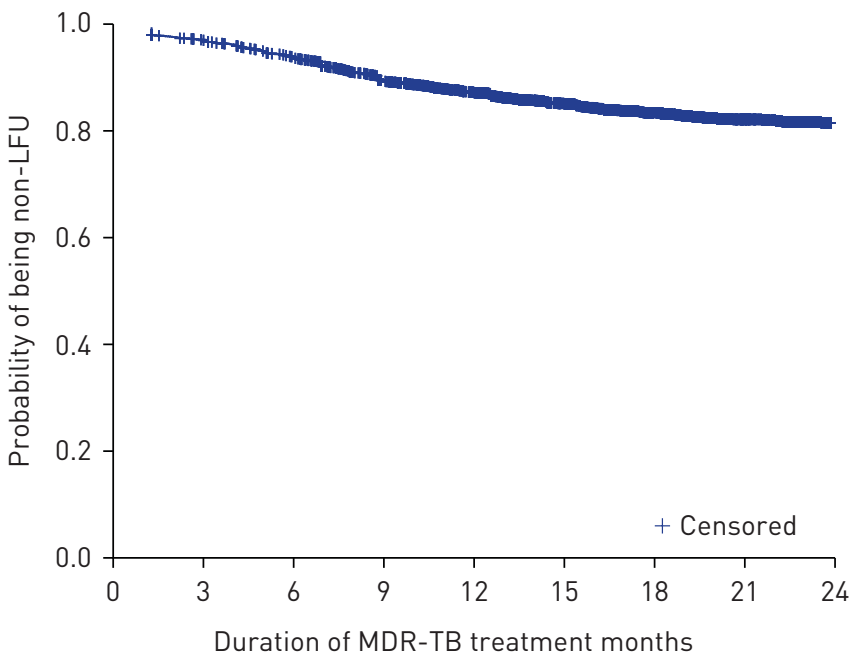

At risk $n$ :

$\begin{array}{lllllllll}4099 & 3921 & 3731 & 3322 & 3051 & 2612 & 2155 & 1456 & 1004\end{array}$ 
FIGURE 3 Time to loss to follow-up (LFU) after starting multidrugresistant tuberculosis (MDR-TB) treatment by national income category, using Kaplan-Meier analysis.

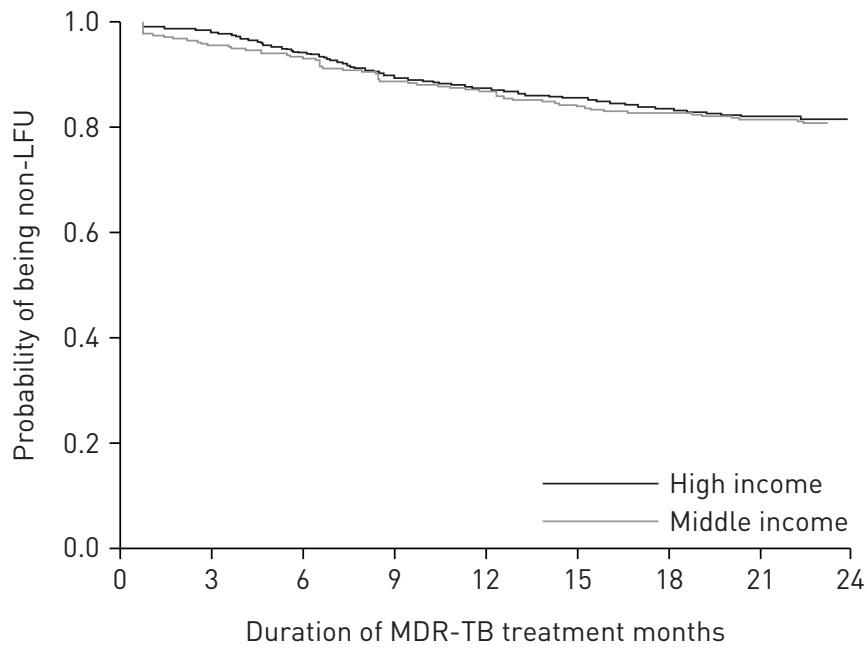

The timing of LFU in our study (comprising treatment prior to 2008) provides findings from the recent past that are broadly similar to studies reporting more recent treatment cohorts (published during 2006-2015) [7-16], where the majority of LFU tended also to be in the initial intensive phase of treatment (see Introduction). Further research, similar to our study, on aggregated datasets from more recent cohorts (and particularly cohorts using the new 9-11-month regimens) would help to identify if this pattern of the majority of LFU occurring in the early stages of treatment has continued. As research in this area grows, our findings form an important historical context.

Our analysis shows that having a co-infection of HIV is a risk factor for LFU. Historically, poor access to antiretroviral treatment and the lack of coordination between HIV and TB treatment programmes have been highlighted as factors that lead to worse outcomes for those co-infected [21]. Treatment approaches in HIV and TB differ significantly, which can lead to confusion for patients seeking to navigate these divergent systems of care [22]. In some cases this leads to preferential adherence of HIV treatment over MDR-TB treatment due to increased tolerability and commitment to HIV treatment and a reduced pill burden [23]. This can create further barriers to effective coordination of treatment with patients comorbidly infected with HIV and MDR-TB.

Better treatment outcomes in children compared with adults have been reported in co-infected HIV populations [24] and non-HIV populations [25]. Our analysis identified an increased risk of LFU in those

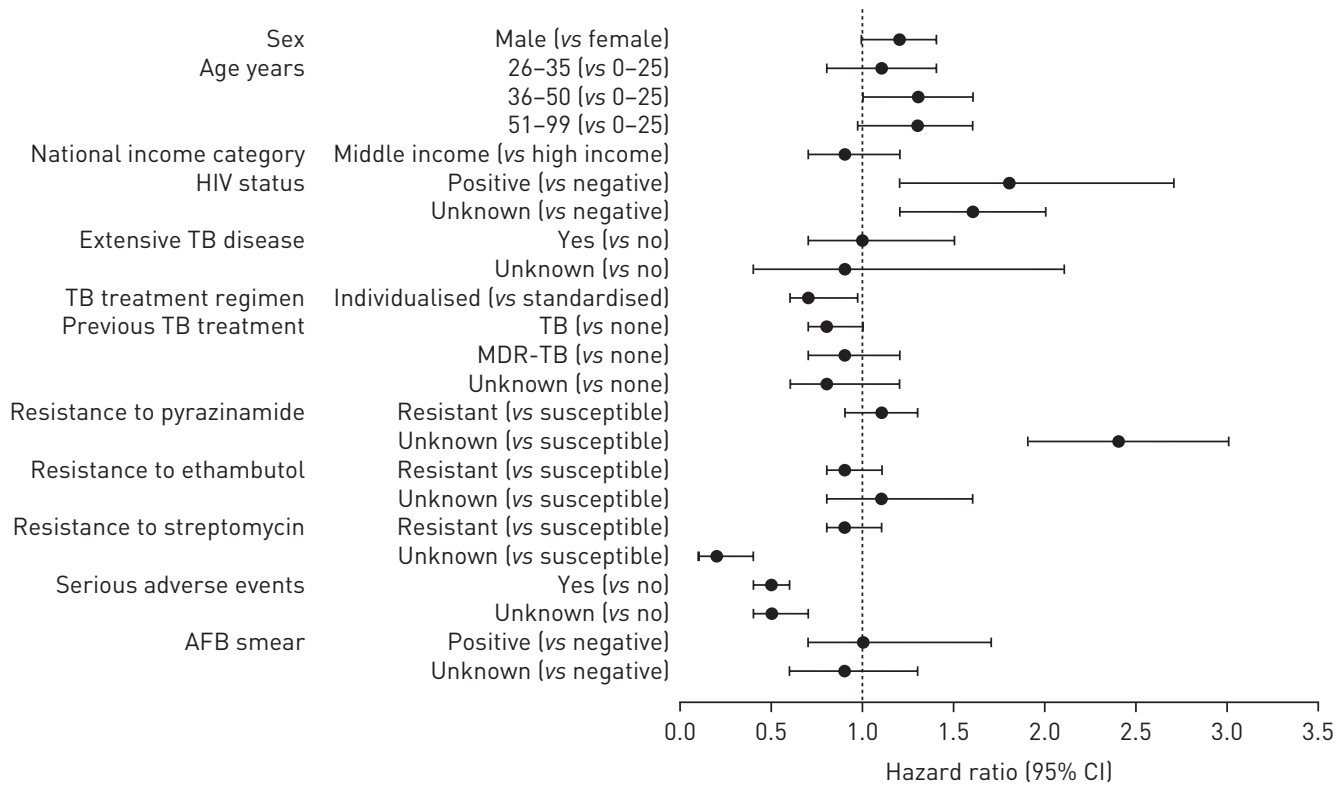

FIGURE 4 Adjusted hazard ratios of risk factors associated with loss to follow-up among multidrug-resistant tuberculosis (MDR-TB) patients ( $\mathrm{n}=4099$ ). AFB: acid-fast bacilli. 
aged 36-50 years (versus $0-25$ years). This could be explained by increased family support offered to younger family members with MDR-TB. Furthermore, the competing demands of employment and dependents for individuals in the working-age groups could also be influential in their greater risk of LFU, although an increase in LFU was not seen on those aged 26-35 years [24].

The finding that patients with a reported serious adverse event had an decreased risk of LFU appears counter-intuitive, as one might predict that these experiences increase the likelihood that patients will stop tolerating treatment and disengage. Indeed, previous studies identified that serious adverse events increased the risk of LFU $[10,13]$. However, a plausible counter-argument exists that those with a recorded serious adverse event are those who are taking medication, and therefore are more likely to experience a serious adverse event due to their greater adherence and have it recorded due to their engagement with services. This strong engagement with services may reduce their likelihood of becoming LFU. The differing findings of previous studies and our findings here could also be explained by differing recording practices of adverse events.

For several variables in our Cox proportional hazards model (HIV status, additional resistance to pyrazinamide, additional resistance to streptomycin and presence of serious adverse event), the "unknown" category was significantly associated with being LFU. Weaker TB treatment programmes may be the confounding factor, where poorer recording practices and limited efforts at following up patients are both associated with weaker programmes.

The study has several limitations. The large number (55\%) of excluded patient records may have introduced bias in our findings. Excluding data with an outcome of death could lead to an overestimation as those that died are not counted in the denominator. Missing data could lead to a potential underestimation of LFU where these missing records may be due to LFU but not recorded as such. Excluding data of those not receiving even the first month of treatment leads to an underestimation of LFU as they are not counted in those identified as LFU. This could also have led to an artefactual prolongation of LFU as patients who would have been early interrupters were selectively removed from the cohorts. The direction of bias from the excluded cohorts is unclear, although the overall rate of LFU is lower than in the main dataset, indicating our overall rate may be an underestimate. The data quality was variable, although attempts have been made to ensure the dataset is as complete as possible. It is possible there are artefactual influences on our dataset that we cannot identify. In addition, we did not have data available for some patient variables that we were interested in, such as comorbid substance misuse or treatment interruptions of $<2$ consecutive months [26]. The cohorts also do not contain valid measures of the practice of directly observed treatment, which is closely associated with treatment outcomes among MDR-TB patients [27]. Furthermore, there are likely to be other types of programme-related or treatment-related risk factors associated with LFU that we did not have data for, such as the efforts of programmes to engage with patients with interrupted treatments.

Given the high proportion of excluded data, caution is needed when generalising from our findings. In addition, the cohorts we included all pre-date 2008 so these findings may not generalise to current programme management of MDR-TB, particularly for patients treated with the recent standardised 9-11-month regimen [20]. This shorter regimen has the potential to reduce LFU, although since $75 \%$ of LFU in our dataset occurred by 11 months, LFU may only reduce by a quarter if the timing of LFU is similar to that in our dataset.

Although some treatment cohorts in this dataset were from resource-constrained contexts, none were from low-income countries. Our findings may not therefore generalise to these settings. We have identified risk factors that are associated with LFU; further work needs to be done to explore the mechanisms that drive stubbornly high rates of LFU in all MDR-TB programmes, including in low-income settings. For instance, the presence of comorbid depression may reduce adherence in MDR-TB as it does in other health conditions $[28,29]$.

One potential use of the findings of our study for treatment programmes is the development of an evidence-based risk assessment tool for identifying patients at increased risk of LFU. Findings from our study and other aggregated or local studies could inform the development of such a tool. The consideration of certain patient-related and programmatic risk factors (such as those identified in this study) could assist in the identification of patients at high risk of becoming LFU during treatment and prompt proactive interventions to prevent this from occurring.

\section{Conclusions}

The call within the global End TB Strategy of patient-centred care should be pursued to address the ongoing issue of LFU. Our findings suggest that MDR-TB treatment programmes could offer targeted, enhanced support to prevent LFU in those of working age and patients with HIV co-infection. Further 
research examining the timing of LFU in more recent treatment cohorts would add to our knowledge of this important aspect of MDR-TB treatment.

Acknowledgements: We would like to thank all the members of the Collaborative Group for Meta-Analysis of Individual Patient Data who allowed us to use their data in this study. D. Falzon is an employee of the World Health Organization (WHO); he is responsible for the views expressed in this publication and they do not necessarily represent the decisions or policies of the WHO. The designations used and the presentation of the material in this publication do not imply the expression of any opinion whatsoever on the part of the WHO concerning the legal status of any country, territory, city or area, or of its authorities, or concerning the delimitation of its frontiers or boundaries.

Conflict of interest: I.F. Walker reports grants from DFID, UK Government, during the conduct of the study. O. Shi has nothing to disclose. J.P. Hicks has nothing to disclose. H. Elsey has nothing to disclose. X. Wei has nothing to disclose. D. Menzies has nothing to disclose. Z. Lan has nothing to disclose. D. Falzon has nothing to disclose. G.B. Migliori has nothing to disclose. C. Pérez-Guzmán has nothing to disclose. M.H. Vargas has nothing to disclose. L. García-García has nothing to disclose. J. Sifuentes Osornio has nothing to disclose. A. Ponce-De-León has nothing to disclose. M. van der Walt has nothing to disclose. J.M. Newell has nothing to disclose.

Support statement: Funding for this analysis was provided by COMDIS-HSD, a research consortium funded by UK aid from the UK government; however, the views expressed do not necessarily reflect the UK government's official policies. The funder had no bearing on the study findings nor did they approve or censor the manuscript. Funding information for this article has been deposited with the Crossref Funder Registry.

\section{References}

1 Ahuja SD, Ashkin D, Avendano M, et al. Multidrug resistant pulmonary tuberculosis treatment regimens and patient outcomes: an individual patient data meta-analysis of 9,153 patients. PLoS Med 2012; 9: e1001300.

2 Falzon D, Gandhi N, Migliori GB, et al. Resistance to fluoroquinolones and second-line injectable drugs: impact on MDR-TB outcomes. Eur Respir J 2013; 42: 156-168.

3 Migliori GB, Sotgiu G, Gandhi NR, et al. Drug resistance beyond XDR-TB: results from a large individual patient data meta-analysis. Eur Respir J 2013; 42: 169-179.

4 World Health Organization. Definitions and Reporting Framework for Tuberculosis - 2013 Revision (WHO/ HTM/TB/2013.2). Geneva, WHO, 2013.

$5 \quad$ World Health Organization. Global Tuberculosis Report 2017. Geneva, WHO, 2017.

6 Johnston JC, Shahidi NC, Sadatsafavi M, et al. Treatment outcomes of multidrug-resistant tuberculosis: a systematic review and meta-analysis. PLoS One 2009; 4: e6914.

7 Cox HS, Kalon S, Allamuratova S, et al. Multidrug-resistant tuberculosis treatment outcomes in Karakalpakstan, Uzbekistan: treatment complexity and XDR-TB among treatment failures. PLoS One 2007; 2: e1126.

8 Holtz T, Lancaster J, Laserson K, et al. Risk factors associated with default from multidrug-resistant tuberculosis treatment, South Africa, 1999-2001. Int J Tuberc Lung Dis 2006; 10: 649-655.

9 Miller A, Gelmanova I, Keshavjee S, et al. Alcohol use and the management of multidrug-resistant tuberculosis in Tomsk, Russian Federation. Int J Tuberc Lung Dis 2012; 16: 891-896.

10 Tupasi TE, Garfin AM, Kurbatova EV, et al. Factors associated with loss to follow-up during treatment for multidrug-resistant tuberculosis, the Philippines, 2012-2014. Emerging Infect Dis 2016; 22: 491-502.

11 Kuchukhidze G, Kumar AM, de Colombani P, et al. Risk factors associated with loss to follow-up among multidrug-resistant tuberculosis patients in Georgia. Public Health Action 2014; 4: Suppl. 2, S41-S46.

12 Lalor MK, Greig J, Allamuratova S, et al. Risk factors associated with default from multi- and extensively drug-resistant tuberculosis treatment, Uzbekistan: a retrospective cohort analysis. PLoS One 2013; 8: e78364.

13 Sanchez-Padilla E, Marquer C, Kalon S, et al. Reasons for defaulting from drug-resistant tuberculosis treatment in Armenia: a quantitative and qualitative study. Int J Tuberc Lung Dis 2014; 18: 160-167.

14 Shringarpure KS, Isaakidis P, Sagili KD, et al. Loss-to-follow-up on multidrug resistant tuberculosis treatment in Gujarat, India: the WHEN and WHO of it. PLoS One 2015; 10: e0132543.

15 Farley JE, Ram M, Pan W, et al. Outcomes of multi-drug resistant tuberculosis (MDR-TB) among a cohort of South African patients with high HIV prevalence. PLoS One 2011; 6: e20436.

16 Javaid A, Shaheen Z, Shafqat M, et al. Risk factors for high death and loss-to-follow-up rates among patients with multidrug-resistant tuberculosis at a programmatic management unit. Am J Infect Control 2017; 45: 190-193.

17 Laserson K, Thorpe L, Leimane V, et al. Speaking the same language: treatment outcome definitions for multidrug-resistant tuberculosis. Int J Tuberc Lung Dis 2005; 9: 640-645.

18 The World Bank. DataBank: World Development Indicators. 2018. http://databank.worldbank.org/data/source/ world-development-indicators Date last accessed: April 19, 2019.

19 World Health Organization. Global Tuberculosis Report 2015. Geneva, WHO, 2015.

20 Khan FA, Salim MH, du Cros P, et al. Effectiveness and safety of standardised shorter regimens for multidrug-resistant tuberculosis: individual patient data and aggregate data meta-analyses. Eur Respir J 2017; 50: 1700061.

21 Wells CD, Cegielski JP, Nelson LJ, et al. HIV infection and multidrug-resistant tuberculosis - the perfect storm. J Infect Dis 2007; 196: Suppl. 1, S86-S107.

22 Daftary A, Calzavara L, Padayatchi N. The contrasting cultures of HIV and tuberculosis care. AIDS 2015; 29: 1-4.

23 Daftary A, Padayatchi N, O’Donnell M. Preferential adherence to antiretroviral therapy over tuberculosis (TB) treatment: a qualitative study of drug-resistant TB/HIV co-infected patients in South Africa. Glob Public Health 2014; 9: 1107-1116.

24 Isaakidis P, Casas E, Das M, et al. Treatment outcomes for HIV and MDR-TB co-infected adults and children: systematic review and meta-analysis. Int J Tuberc Lung Dis 2015; 19: 969-978.

25 Ettehad D, Schaaf HS, Seddon JA, et al. Treatment outcomes for children with multidrug-resistant tuberculosis: a systematic review and meta-analysis. Lancet Infect Dis; 12: 449-456. 
26 Wei XL, Yin J, Zou GY, et al. Treatment interruption and directly observed treatment of multidrug-resistant tuberculosis patients in China. Int J Tuberc Lung Dis 2015; 19: 413-419.

27 Yin J, Yuan J, Hu Y, et al. Association between directly observed therapy and treatment outcomes in multidrug-resistant tuberculosis: a systematic review and meta-analysis. PLoS One 2016; 11: e0150511.

28 Walker IF, Baral SC, Wei X, et al. Multidrug-resistant tuberculosis treatment programmes insufficiently consider comorbid mental disorders. Int J Tuberc Lung Dis 2017; 21: 603-609.

29 Kardas P, Lewek P, Matyjaszczyk M. Determinants of patient adherence: a review of systematic reviews. Front Pharmacol 2013; 4: 91. 